\title{
Lorentz transformations via Pauli matrices
}

Z. Ahsan ${ }^{1}$, J. López-Bonilla ${ }^{2}$, B. Man Tuladhar ${ }^{3}$,

${ }^{1}$ Dept. of Mathematics, Aligarh Muslim University, Aligarh 202 002, Aligarh, India

${ }^{2}$ ESIME-Zacatenco, Instituto Politécnico Nacional,

Edif. 5, 1er. Piso, Col. Lindavista 07738, México DF; jlopezb@ipn.mx

${ }^{3}$ Kathmandu University, Dhulikhel, Kavre, Nepal

Abstract.- We exhibit expressions, in terms of Pauli matrices, which directly generate Lorentz transformations in Minkowski space.

Key words.- Pauli matrices, Lorentz transformations, Infeld-van der Waerden symbols

\section{Council for Innovative Research}

Peer Review Research Publishing System

Journal of Advances in Natural Sciences

Vol. 2, No. 1

editorjansonline@gmail.com

www.cirworld.com 
In space time an event is represented by $\left(x^{j}\right)=(c t, x, y, z), j=0, \ldots, 3$, with the metric $\left(g_{j r}\right)=\operatorname{Diag}(1,-1,-1,-1)$. If it is necessary to employ another frame of reference, then the new coordinates $\tilde{x}^{r}$ are connected with $x^{j}$ via the linear transformation:

$$
\tilde{x}^{j}=L_{r}^{j} x^{r}
$$

where the Lorentz matrix $\underset{\sim}{L}$ verifies the restriction :

$$
L^{j}{ }_{a} g_{r j} L_{b}^{r}=g_{a b}
$$

because the Minkowskian line element must remain invariant under $\underset{\sim}{L}$, that is, $\tilde{x}^{r} \tilde{x}_{r}=x^{r} x_{r}$.

From (2) we see that $\underset{\sim}{L}$ has six degrees of freedom, which permits to work with four complex numbers $\alpha, \beta, \gamma, \delta$ such that $\alpha \delta-\beta \gamma=1$, then the components of homogeneous Lorentz transformation $\underset{\sim}{L}$ can be written in the form [1-4]:

$$
\begin{aligned}
& L_{0}^{0}=\frac{1}{2}\left(\alpha \alpha^{*}+\beta \beta^{*}+\gamma \gamma^{*}+\delta \delta^{*}\right), \\
& L_{1}^{0}=\frac{1}{2}\left(\alpha^{*} \beta+\gamma^{*} \delta\right)+\text { c.c. }, \\
& L_{2}^{0}=\frac{i}{2}\left(\alpha^{*} \beta+\gamma^{*} \delta\right)+\text { c.c. }, \\
& L_{3}^{0}=\frac{1}{2}\left(\alpha \alpha^{*}-\beta \beta^{*}+\gamma \gamma^{*}-\delta \delta^{*}\right) \text {, } \\
& L_{0}^{1}=\frac{1}{2}\left(\alpha^{*} \gamma+\beta^{*} \delta\right)+\text { c.c. }, \\
& L_{1}^{1}=\frac{1}{2}\left(\alpha^{*} \delta+\beta \gamma^{*}\right)+\text { c.c. } \\
& L_{2}^{1}=\frac{i}{2}\left(\alpha^{*} \delta+\beta \gamma^{*}\right)+\text { c.c. }, \\
& L_{3}^{1}=\frac{1}{2}\left(\alpha^{*} \gamma-\beta^{*} \delta\right)+\text { c.c. }, \\
& L_{0}^{2}=\frac{i}{2}\left(\alpha \gamma^{*}-\beta^{*} \delta\right)+\text { c.c. , } \\
& L_{1}^{2}=\frac{i}{2}\left(\alpha \delta^{*}+\beta \gamma^{*}\right)+\text { c.c. }, \\
& L_{2}^{2}=\frac{1}{2}\left(\alpha^{*} \delta-\beta^{*} \gamma\right)+\text { c.c. , } \\
& L_{3}^{2}=\frac{i}{2}\left(\alpha \gamma^{*}+\beta^{*} \delta\right)+\text { c.c. }, \\
& L_{0}^{3}=\frac{1}{2}\left(\alpha \alpha^{*}+\beta \beta^{*}-\gamma \gamma^{*}-\delta \delta^{*}\right) \text {, } \\
& L_{1}^{3}=\frac{1}{2}\left(\alpha^{*} \beta-\gamma^{*} \delta\right)+\text { c.c. , } \\
& L_{2}^{3}=\frac{i}{2}\left(\alpha^{*} \beta-\gamma^{*} \delta\right)+\text { c.c. }, \\
& L^{3}{ }_{3}=\frac{1}{2}\left(\alpha \alpha^{*}-\beta \beta^{*}-\gamma \gamma^{*}+\delta \delta^{*}\right),
\end{aligned}
$$

where c.c. means the complex conjugate of all the previous terms. Therefore, any complex $2 \times 2$ matrix [4-7]:

$$
\left.\bigcup \begin{array}{ll}
\alpha & \beta \\
\gamma & \delta
\end{array}\right), \quad \text { Det } \underset{\sim}{\bigcup}=\alpha \delta-\beta \gamma=1 \text {, }
$$

generates a Lorentz matrix via (3).

The following relations, which are not explicitly in the literature, give us directly all the components (3): 


$$
\begin{gathered}
L^{\mu}{ }_{v}=-\frac{1}{2} \bigcup^{a r} \sigma^{\mu}{ }_{a j} \sigma_{v b r} U^{\dagger} b j \quad, \quad \mu, v=1,2,3 \\
L^{\mu}{ }_{0}=\frac{1}{2} \sigma^{\mu}{ }_{j r} \mathrm{Q}^{j r} \quad, \quad \mu=0, \ldots, 3, \quad L_{v}^{0}=-\frac{1}{2} \sigma_{v j k} \mathrm{R}^{j k} \quad, \quad v=1,2,3
\end{gathered}
$$

such that:

$$
\bigcup^{\dagger}=\left(\begin{array}{ll}
\alpha^{*} & \gamma^{*} \\
\beta^{*} & \delta^{*}
\end{array}\right), \quad \underset{\sim}{\mathrm{Q}}=\bigcup_{\sim}^{\bigcup} \bigcup^{\dagger}, \quad \underset{\sim}{\mathrm{R}}=\bigcup_{\sim}^{\dagger} \bigcup_{\sim},
$$

with the Infeld-van der Waerden symbols [8-11]:

$$
\begin{aligned}
& \left(\sigma_{a b}^{0}\right)=\left(\sigma_{0 a b}\right)=I=\left(\begin{array}{ll}
1 & 0 \\
0 & 1
\end{array}\right), \quad\left(\sigma_{a b}^{1}\right)=\left(-\sigma_{1 a b}\right)=\sigma_{x}=\left(\begin{array}{ll}
0 & 1 \\
1 & 0
\end{array}\right) \\
& \left(\sigma_{a b}^{2}\right)=\left(-\sigma_{2 a b}\right)=-\sigma_{y}=\left(\begin{array}{ll}
0 & \mathrm{i} \\
-\mathrm{i} & 0
\end{array}\right), \quad\left(\sigma_{a b}^{3}\right)=\left(-\sigma_{3 a b}\right)=\sigma_{z}=\left(\begin{array}{cc}
1 & 0 \\
0 & -1
\end{array}\right)
\end{aligned}
$$

where $\sigma_{j}, j=x, y, z$ are the known Cayley-Sylvester-Pauli matrices $[4,6,12-14]$.

The expressions (5) show explicitly a direct relationship between $\underset{\sim}{L}$ and $U$, which may be useful in applications of spinorial calculus [11] in Minkowski spacetime.

\section{References}

1. J. Aharoni, The special theory of relativity, Clarendon Press, Oxford (1959)

2. J. L. Synge, Relativity: the special theory, North-Holland Pub., Amsterdam (1965)

3. J. López-Bonilla, J. Morales, G. Ovando, On the homogeneous Lorentz transformation, Bull. Allahabad Math. Soc. 17 (2002) 53-58

4. J. López-Bonilla, J. Morales, G. Ovando, Quaternions, 3-rotations and Lorentz transformations, Indian J. Theor. Phys. 52, No. 2 (2004) 91-96

5. J. L. Synge, Quaternions, Lorentz transformations, and the Conway-Dirac- Eddington matrices, Comm. Dublin Inst. Adv. Stud. A 21 (1972) 1-67

6. L. H. Ryder, Quantum field theory, Cambridge University Press (1985)

7. I. Guerrero, J. López-Bonilla, L. Rosales, Rotations in three and four dimensions via $2 \times 2$ complex matrices and quaternions, The Icfai Univ. J. Phys. 1, No. 2 (2008) 7-13

8. B. L. van der Waerden, Spinoranalyse, Nachr. Ges. Wiss. Göttingen Math.-Phys. (1929) 100-109

9. L. Infeld, Die verallgemeinerte spinorenrechnung und die Diracschen gleichungen, Phys. Z. 33 (1932) 475-483

10. L. Infeld, B. L. van der Waerden, Die wellengleichung des elektrons in der allgemeinen relativitätstheorie, Sitz. Ber. Preuss. Akad. Wiss. Physik-Math. KI 9 (1933) 380-401

11. P. O'Donnell, Introduction to 2-spinors in general relativity, World Scientific, Singapore (2003)

12. A. Cayley, A memoir on the theory of matrices, London Phil. Trans. 148 (1858) 17-37

13. J. Sylvester, On quaternions, nonions and sedenions, Johns Hopkins Circ. 3 (1884) 7-9

14. W. Pauli, Zur quantenmechanik des magnetischen elektrons, Zeits f. Physik 43 (1927) 601-623 